\title{
Attractor-Based Simultaneous Design of the Minimum Set of Control Nodes and Controllers in Boolean Networks
}

\author{
Koichi Kobayashi \\ Graduate School of Information Science and Technology, Hokkaido University, Sapporo, Japan \\ Email: k-kobaya@ssi.ist.hokudai.ac.jp
}

Received 20 June 2016; accepted 15 August 2016; published 18 August 2016

Copyright (C) 2016 by author and Scientific Research Publishing Inc.

This work is licensed under the Creative Commons Attribution International License (CC BY). http://creativecommons.org/licenses/by/4.0/

c) (i) Open Access

\begin{abstract}
Design of control strategies for gene regulatory networks is a challenging and important topic in systems biology. In this paper, the problem of finding both a minimum set of control nodes (control inputs) and a controller is studied. A control node corresponds to a gene that expression can be controlled. Here, a Boolean network is used as a model of gene regulatory networks, and control specifications on attractors, which represent cell types or states of cells, are imposed. It is important to design a gene regulatory network that has desired attractors and has no undesired attractors. Using a matrix-based representation of BNs, this problem can be rewritten as an integer linear programming problem. Finally, the proposed method is demonstrated by a numerical example on a WNT5A network, which is related to melanoma.
\end{abstract}

\section{Keywords}

Boolean Networks, Integer Linear Programming, Minimum Set of Control Nodes, Singleton Attractors

\section{Introduction}

Modeling, analysis, and control of gene regulatory networks are one of the fundamental problems in the field of systems biology. Control of gene regulatory networks is closely related to therapeutic interventions, which are realized by radiation, chemotherapy, and so on. Hence, developing control theory of gene regulatory networks is important for gene therapy technologies (see, e.g., [1]) in the future. When we discuss the control problem of gene regulatory networks, we frequently assume that some of genes can be arbitrarily controlled. In this paper, such genes are called a control node. Since in mathematical models of gene regulatory networks, control nodes 
are not necessarily specified, the problem of finding a set of control nodes based on control specifications is important as a kind of inverse problems. Needless to say, it is desirable that the number of control nodes is small.

In this paper, we study simultaneous design of a minimum set of control nodes and controllers in a Boolean networks (BN). A BN [2] is one of the simplified models such as Bayesian networks and piecewise affine systems [3], and is widely used in theoretical analysis of gene regulatory networks (see, e.g., [4]-[19]). In the BN, dynamics such as interactions between genes are expressed by Boolean functions, that is, gene expression is expressed by a binary value (0 or 1). As a generalized version of BNs, a probabilistic BN has been proposed in [20]. In the simultaneous design problem studied in this paper, control specifications on attractors are imposed. Attractors represent cell types or states of cells [21]. In particular, we focus on a singleton attractor, which is also called a fixed point. By solving this problem, we can obtain a gene regulatory network that has desired attractors and has no undesired attractors. Furthermore, we can obtain time evolution (i.e., a Boolean function) of a control node, i.e., a controller by solving this problem. Thus, a solution of the simultaneous design problem provides a helpful suggestion to e.g., design of therapeutic interventions.

As a related problem, the conventional controllability problem of BNs has been widely studied (see, e.g., [7] [12] [13] [17] [18] [22] [23]). However, the problem of finding a minimum set of control nodes has not been considered. In [24], using piecewise affine systems, the problem of choosing a control node has been studied. However, the minimization problem of the number of control nodes has not been considered.

In order to solve the simultaneous design problem, a matrix-based representation of BNs [10] is utilized. Using this representation, this problem can be rewritten as an integer linear programming problem. We may use a matrix-based representation using the semi-tensor product (STP) method [7] [25]. Since manipulation of matrices using the STP is not needed in this paper, we utilize a simple matrix-based representation using truth tables. The effectiveness of the proposed method is presented by an example on a WNT5A network, which is related to melanoma.

Notation: For the finite set $\mathcal{A}$, let $|\mathcal{A}|$ denote the number of elements in $\mathcal{A}$. For the $n$-dimensional vector

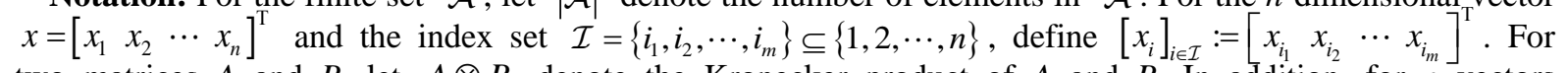
two matrices $A$ and $B$, let $A \otimes B$ denote the Kronecker product of $A$ and $B$. In addition, for $q$ vectors $y_{1}, y_{2}, \cdots, y_{q}$ and the index set $\mathcal{J}=\left\{j_{1}, j_{2}, \cdots, j_{p}\right\} \subseteq\{1,2, \cdots, q\}$, define $\otimes_{j \in \mathcal{J}} y_{j}:=y_{j_{1}} \otimes y_{j_{2}} \otimes \cdots \otimes y_{j_{p}}$. For example, for $q$ two-dimensional vectors $z_{1}, z_{2}, \cdots, z_{q}$ and $\mathcal{J}=\{1,5\}$, we can obtain

$$
\bigotimes_{j \in \mathcal{J}} z_{j}=\left[\begin{array}{l}
z_{1} \\
z_{5}
\end{array}\right]=\left[\begin{array}{c}
z_{1}^{(1)} z_{5}^{(1)} \\
z_{1}^{(1)} z_{5}^{(2)} \\
z_{1}^{(2)} z_{5}^{(1)} \\
z_{1}^{(2)} z_{5}^{(2)}
\end{array}\right],
$$

where $z_{j}^{(i)}$ is the $i$-th element of $z_{j}$. Finally, let $1_{m \times n}$ denote the $m \times n$ matrix whose elements are all one.

\section{Boolean Networks}

Consider the following Boolean network (BN):

$$
\left\{\begin{array}{c}
x_{1}(k+1)=f_{1}\left(\left[x_{j}(k)\right]_{j \in \mathcal{N}_{1}}\right), \\
x_{2}(k+1)=f_{2}\left(\left[x_{j}(k)\right]_{j \in \mathcal{N}_{2}}\right), \\
\vdots \\
x_{n}(k+1)=f_{n}\left(\left[x_{j}(k)\right]_{j \in \mathcal{N}_{n}}\right),
\end{array}\right.
$$

where $\quad x=\left[\begin{array}{llll}x_{1} & x_{2} & \cdots & x_{n}\end{array}\right]^{\mathrm{T}} \in\{0,1\}^{n}$ is the state (e.g., the expression of genes), and $k \in\{0,1,2, \cdots\}$ is the discrete time. The set $\mathcal{N}_{i} \subseteq\{1,2, \cdots, n\}$ is a given index set, and expresses the adjacency relation of nodes corresponding to genes. The function $f_{i}:\{0,1\}^{\left|\mathcal{N}_{i}\right|} \rightarrow\{0,1\}^{1}$ is a given Boolean function consisting of logical operators such as AND $(\wedge)$, OR $(\vee)$, and NOT $(\neg)$. If $\mathcal{N}_{i}=\varnothing$ holds, then $x_{i}(k+1)$ is uniquely determined as 0 
or 1 .

Next, the notion of attractors is defined as follows.

Definition 1 The state $x(k)$ is called a singleton attractor if $x(k+1)=x(k)$ holds.

Definition 2 The set of states $\{x(k), x(k+1), \cdots, x(k+p-1)\}$ is called a periodic attractor if $x(k+i) \neq x(k)$, $i=1,2, \cdots, p-1$ and $x(k+p)=x(k)$ hold.

We present a simple example.

Example 1 Consider the following $B N$ :

$$
\left\{\begin{array}{l}
x_{1}(k+1)=x_{3}(k), \\
x_{2}(k+1)=x_{1}(k) \wedge \neg x_{3}(k), \\
x_{3}(k+1)=x_{1}(k) \vee \neg x_{2}(k) .
\end{array}\right.
$$

In this $B N, \mathcal{N}_{1}=\{3\}, \mathcal{N}_{2}=\{1,3\}$, and $\mathcal{N}_{3}=\{1,2\}$ hold. Figure 1 shows the state transition diagram. From Figure 1, we see that there exist two singleton attractors and one periodic attractor with $p=2$.

In this paper, we focus on only a set of singleton attractors. Hereafter, let $\mathcal{A}$ denote a set of singleton attractors for the BN (1).

\section{Problem Formulation}

The states $x_{1}, x_{2}, \cdots, x_{n}$ are decomposed to two groups $x_{i}, i \in \mathcal{P}$ and $x_{i}, i \in \mathcal{C}$, where $\mathcal{P}$ and $\mathcal{C}$ are index sets satisfying $\mathcal{P} \cup \mathcal{C}=\{1,2, \cdots, n\}$ and $\mathcal{P} \cap \mathcal{C}=\varnothing$. Then, defining $u_{i}(k):=x_{i}(k), i \in \mathcal{C}$, the BN (1) can be rewritten as

$$
\left\{\begin{array}{l}
x_{i}(k+1)=f_{i}\left(\left[u_{j}(k)\right]_{j \in \mathcal{L}_{i}},\left[u_{j}(k)\right]_{j \in \mathcal{M}_{i}}\right), i \in \mathcal{P}, \\
u_{i}(k+1)=f_{i}\left(\left[u_{j}(k)\right]_{j \in \mathcal{L}_{i}},\left[u_{j}(k)\right]_{j \in \mathcal{M}_{i}}\right), i \in \mathcal{C},
\end{array}\right.
$$

where $\mathcal{L}_{i}, \mathcal{M}_{i}, \mathcal{L}_{i}$, and $\mathcal{M}_{i}$ are an index set. For each $i \in \mathcal{P} \cup \mathcal{C}$, the relations $\mathcal{L}_{i} \cup \mathcal{M}_{i}=\mathcal{N}_{i}$ and $\mathcal{L}_{i} \cap \mathcal{M}_{i}=\varnothing$ hold. The Boolean function $f_{i}, i \in \mathcal{P}$ is given in advance. The Boolean function $f_{i}, i \in \mathcal{C}$ is not given, and must be found. In other words, we consider that some of Boolean functions in the BN (1) are replaced with new Boolean functions. In the case of $\mathcal{C}=\varnothing,(3)$ is the same as (1). Furthermore, in the BN (3), $u_{i}(k)$ corresponds to the control node (i.e., the control input). The Boolean function $f_{i}, i \in \mathcal{C}$ can be regarded as the controller.

Then, consider the following problem.

Problem 1 For the BN (3), suppose that Boolean functions $f_{1}, f_{2}, \cdots, f_{n}$ are given. Suppose also that the set of desired singleton attractors and the set of undesired singleton attractors are given by

$$
\mathcal{A}_{d}=\left\{\alpha_{1}, \alpha_{2}, \cdots, \alpha_{m_{d}}\right\}, \alpha_{i} \in\{0,1\}^{n}
$$

and

$$
\mathcal{A}_{u}=\left\{\beta_{1}, \beta_{2}, \cdots, \beta_{m_{u}}\right\}, \beta_{i} \in\{0,1\}^{n},
$$

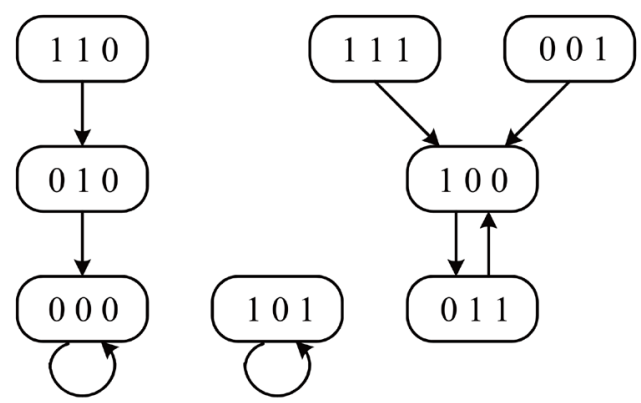

Figure 1. State transition diagram, where the number assigned to each node implies $x_{1}, x_{2}, x_{3}$. 
respectively. Then, find a minimum set of control nodes and a controller $f_{i}, i \in \mathcal{C}$ satisfying the following two conditions:

$$
\mathcal{A}_{d} \subseteq \mathcal{A}
$$

and

$$
\mathcal{A}_{u} \cap \mathcal{A}=\varnothing .
$$

If this problem is infeasible, then there exists no controller that realizes specifications on singleton attractors. This fact implies that the BN (3) is uncontrollable from the viewpoint of singleton attractors, and the change of the graph structure $\mathcal{N}_{i}$ is required. On the other hand, as a solution, $\mathcal{C}=\varnothing$ may be obtained. This solution implies that a given BN has desired singleton attractors and has no undesired singleton attractors. That is, control nodes are not necessary. Thus, Problem 1 can be regarded as a kind of the controllability problems.

If the set $\mathcal{C}$ (i.e., $\mathcal{P}$ ) is given, Problem 1 has been already solved in [10]. Problem 1 is more general than the problem studied in [10]. By solving Problem 1, we can obtain both the minimum set of control nodes and the controller. To the best of our knowledge, such problem has not been studied so far.

\section{Matrix-Based Representation of Boolean Networks}

In order to solve Problem 1, we use a matrix-based representation of BNs [10].

In this representation, one state $x_{i}(k)$ in the BN (1) is expressed by two binary variables $x_{i}^{0}(k)$ and $x_{i}^{1}(k)$. The binary variable $x_{i}^{0}(k)$ is defined by $x_{i}^{0}(k):=1-x_{i}(k)$. The binary variable $x_{i}^{1}(k)$ is defined by $x_{i}^{0}(k):=x_{i}(k)$. Using $x_{i}^{0}(k)$ and $x_{i}^{1}(k)$, define

$$
\bar{x}_{i}(k):=\left[\begin{array}{c}
x_{i}^{0}(k) \\
x_{i}^{1}(k)
\end{array}\right]\left(=\left[\begin{array}{c}
1-x_{i}(k) \\
x_{i}(k)
\end{array}\right]\right) .
$$

Then, the matrix-based representation for $x_{i}(k+1)$ is given by

$$
\bar{x}_{i}(k+1)=A_{i} \bigotimes_{j \in \mathcal{N}_{i}} \bar{x}_{j}(k),
$$

where $A_{i} \in\{0,1\}^{2 \times 2^{\left|N_{i}\right|}}$ and $\otimes_{j \in \mathcal{N}_{i}} \bar{X}_{j}(k) \in\{0,1\}^{||^{\left|\mathcal{N}_{i}\right|}}$. The matrix $A_{i}$ can be derived from truth tables. We present a simple example.

Example 2 Consider the BN (2) again. Then, we can obtain the truth table for each $x_{i}(k+1)$. See Table 1 . From these truth tables, we can obtain the following matrix-based representation:

$$
\begin{aligned}
& {\left[\begin{array}{l}
x_{1}^{0}(k+1) \\
x_{1}^{1}(k+1)
\end{array}\right]=\underbrace{\left[\begin{array}{ll}
1 & 0 \\
0 & 1
\end{array}\right]}_{A_{1}}\left[\begin{array}{c}
x_{3}^{0}(k) \\
x_{3}^{1}(k)
\end{array}\right],} \\
& {\left[\begin{array}{c}
x_{2}^{0}(k+1) \\
x_{2}^{1}(k+1)
\end{array}\right]=\underbrace{\left[\begin{array}{llll}
1 & 1 & 0 & 1 \\
0 & 0 & 1 & 0
\end{array}\right]}_{A_{2}}\left[\begin{array}{l}
x_{1}^{0}(k) x_{3}^{0}(k) \\
x_{1}^{0}(k) x_{3}^{1}(k) \\
x_{1}^{1}(k) x_{3}^{0}(k) \\
x_{1}^{1}(k) x_{3}^{1}(k)
\end{array}\right],}
\end{aligned}
$$

Table 1. Truth tables for $x_{1}(k+1), \quad x_{2}(k+1)$, and $x_{3}(k+1)$.

\begin{tabular}{cccccccc}
\hline$x_{3}(k)$ & $x_{1}(k+1)$ & $x_{1}(k)$ & $x_{3}(k)$ & $x_{2}(k+1)$ & $x_{1}(k)$ & $x_{2}(k)$ & $x_{3}(k+1)$ \\
\hline 0 & 0 & 0 & 0 & 0 & 0 & 0 & 1 \\
1 & 1 & 0 & 1 & 0 & 0 & 1 & 0 \\
& 1 & 0 & 1 & 1 & 0 & 1 \\
& 1 & 1 & 0 & 1 & 1 \\
\hline
\end{tabular}




$$
\left[\begin{array}{c}
x_{3}^{0}(k+1) \\
x_{3}^{1}(k+1)
\end{array}\right]=\underbrace{\left[\begin{array}{llll}
0 & 1 & 0 & 0 \\
1 & 0 & 1 & 1
\end{array}\right]}_{A_{3}}\left[\begin{array}{c}
x_{1}^{0}(k) x_{2}^{0}(k) \\
x_{1}^{0}(k) x_{2}^{1}(k) \\
x_{1}^{1}(k) x_{2}^{0}(k) \\
x_{1}^{1}(k) x_{2}^{1}(k)
\end{array}\right],
$$

where each element of $A_{i}, i=1,2,3$ is given by a binary value ( 0 or 1$)$, and a sum of all elements in each column of $A_{i}$ is equal to 1 . The second row in $A_{i}, i=1,2,3$ corresponds to the column in $x_{i}(k+1)$ in truth tables.

See [10] for further details of deriving the matrix $A_{i}$.

The matrix-based representation is useful for considering Problem 1. Using the matrix-based representation, the problem of finding a Boolean function is reduced to the problem of finding $A_{i}$. The latter problem is relatively easier than the former problem, and can be solved by using some computational tools.

Remark 1 Recently, there have been many results about a matrix-based representation of Boolean functions using the semi-tensor product (STP) of matrices (see, e.g., [7] [25]). Of course, the matrix-based representation using the STP method may be utilized. In this paper, we focus on singleton attractors (i.e., the steady state), and time sequences of the state are not computed. Hence, manipulation of matrices using the STP is not needed, and the simple matrix-based representation using truth tables is utilized.

\section{Solution Method for Problem 1}

Based on the matrix-based representation of BNs explained in the previous section, we consider a solution method for Problem 1.

As preparations, the notation is defined. If $A_{i}$ in (8) includes decision variables, then $A_{i}$ is denoted by $\tilde{A}_{i}$. Let $\alpha_{i}^{(j)}$ and $\beta_{i}^{(j)}$ denote the $j$-th element of $\alpha_{i}$ and $\beta_{i}$ in $\mathcal{A}_{d}$ of (4) and $\mathcal{A}_{u}$ of (5), respectively. Define

$$
\bar{\alpha}_{i}^{(j)}:=\left[\begin{array}{c}
1-\alpha_{i}^{(j)} \\
\alpha_{i}^{(j)}
\end{array}\right], \bar{\beta}_{i}^{(j)}:=\left[\begin{array}{c}
1-\beta_{i}^{(j)} \\
\beta_{i}^{(j)}
\end{array}\right] .
$$

Since only singleton attractors are focused in Problem 1, Problem 1 is equivalently transformed into the following problem.

Problem 2 Find matrices $\tilde{A}_{1}, \tilde{A}_{2}, \cdots, \tilde{A}_{n}$ and binary variables $\delta_{1}, \delta_{2}, \cdots, \delta_{n}$ minimizing the cost function $\sum_{i=1}^{n} \delta_{i}$ subject to the following two conditions. (i) For each $i \in\left\{1,2, \cdots, m_{d}\right\}$, the following constraints corresponding to (6) are satisfied:

$$
\begin{gathered}
\bar{\alpha}_{i}^{(1)}=\left\{\left(1-\delta_{1}\right) A_{1}+\delta_{1} \tilde{A}_{1}\right\} \underset{j \in \mathcal{N}_{1}}{\bigotimes} \bar{\alpha}_{i}^{(j)}, \\
\bar{\alpha}_{i}^{(2)}=\left\{\left(1-\delta_{2}\right) A_{2}+\delta_{2} \tilde{A}_{2}\right\} \underset{j \in \mathcal{N}_{2}}{\bigotimes} \bar{\alpha}_{i}^{(j)}, \\
\vdots \\
\bar{\alpha}_{i}^{(n)}=\left\{\left(1-\delta_{n}\right) A_{n}+\delta_{n} \tilde{A}_{n}\right\} \underset{j \in \mathcal{N}_{n}}{\bigotimes} \bar{\alpha}_{i}^{(j)} .
\end{gathered}
$$

(ii) For each $i \in\left\{1,2, \cdots, m_{u}\right\}$, at least one of the following constraints is satisfied:

$$
\begin{gathered}
\bar{\beta}_{i}^{(1)} \neq\left\{\left(1-\delta_{1}\right) A_{1}+\delta_{1} \tilde{A}_{1}\right\} \underset{j \in \mathcal{N}_{1}}{\otimes} \bar{\beta}_{i}^{(j)}, \\
\bar{\beta}_{i}^{(2)} \neq\left\{\left(1-\delta_{2}\right) A_{2}+\delta_{2} \tilde{A}_{2}\right\} \underset{j \in \mathcal{N}_{2}}{\otimes} \bar{\beta}_{i}^{(j)}, \\
\vdots \\
\bar{\beta}_{i}^{(n)} \neq\left\{\left(1-\delta_{n}\right) A_{n}+\delta_{n} \tilde{A}_{n}\right\} \underset{j \in \mathcal{N}_{n}}{\otimes} \bar{\beta}_{i}^{(j)} .
\end{gathered}
$$

In addition, each element of $\tilde{A}_{1}, \tilde{A}_{2}, \cdots, \tilde{A}_{n}$ takes a binary value, and a sum of each column of these matrices 
is equal to 1 .

In this problem, the condition (i) guarantees that $x(k+1)=x(k)=\alpha_{i}, i=1,2, \cdots, m_{d}$, that is, $\alpha_{i}$, $i=1,2, \cdots, m_{d}$ are included in the set of singleton attractors. The condition (ii) guarantees that $\beta_{i}, i=1,2, \cdots, m_{d}$ are not a singleton attractor. If $\delta_{i}=0, i=1,2, \cdots, n$ are obtained as a solution, then this solution implies $\mathcal{C}=\varnothing$, that is, the controller is not needed. If $\delta_{i}=1, i=1,2, \cdots, n$ are obtained as a solution, then this solution implies $\mathcal{P}=\varnothing$, that is, all Boolean functions are changed.

Problem 2 can be rewritten as an integer linear programming (ILP) problem, and can be solved by a suitable solver. An SMT (Satisfiability Modulo Theories) solver such as the Yices SMT Solver [26] can also be applied to Problem 2.

Here, using a simple example, we explain a method to rewrite Problem 2 as an ILP problem. Consider the BN in Example 2 of Section 4. Then, the constraints appeared in Problem 2 are given by

$$
\begin{aligned}
& {\left[\begin{array}{c}
1-\alpha_{1}^{(1)} \\
\alpha_{1}^{(1)}
\end{array}\right]=\left\{\left(1-\delta_{1}\right)\left[\begin{array}{ll}
0 & 1 \\
1 & 0
\end{array}\right]+\delta_{1}\left[\begin{array}{cc}
1-a_{11} & 1-a_{12} \\
a_{11} & a_{12}
\end{array}\right]\right\}\left[\begin{array}{c}
1-\alpha_{1}^{(2)} \\
\alpha_{1}^{(2)}
\end{array}\right],} \\
& {\left[\begin{array}{c}
1-\alpha_{1}^{(2)} \\
\alpha_{1}^{(2)}
\end{array}\right]=\left\{\left(1-\delta_{2}\right)\left[\begin{array}{ll}
1 & 0 \\
0 & 1
\end{array}\right]+\delta_{2}\left[\begin{array}{cc}
1-a_{21} & 1-a_{22} \\
a_{21} & a_{22}
\end{array}\right]\right\}\left[\begin{array}{c}
1-\alpha_{1}^{(1)} \\
\alpha_{1}^{(1)}
\end{array}\right] \text {, }} \\
& {\left[\begin{array}{c}
1-\alpha_{1}^{(3)} \\
\alpha_{1}^{(3)}
\end{array}\right]=\left\{\left(1-\delta_{3}\right)\left[\begin{array}{llll}
1 & 1 & 0 & 1 \\
0 & 0 & 1 & 0
\end{array}\right]+\delta_{3}\left[\begin{array}{cccc}
1-a_{31} & 1-a_{32} & 1-a_{33} & 1-a_{34} \\
a_{31} & a_{32} & a_{33} & a_{34}
\end{array}\right]\right\} \underbrace{\left[\begin{array}{c}
\left(1-\alpha_{1}^{(1)}\right)\left(1-\alpha_{1}^{(2)}\right) \\
\left(1-\alpha_{1}^{(1)}\right) \alpha_{1}^{(2)} \\
\alpha_{1}^{(1)}\left(1-\alpha_{1}^{(2)}\right) \\
\alpha_{1}^{(1)} \alpha_{1}^{(2)}
\end{array}\right]}_{\bar{\alpha}_{1}^{(1)} \otimes \bar{\alpha}_{1}^{(2)}}}
\end{aligned}
$$

and

$$
\begin{aligned}
& {\left[\begin{array}{c}
1-\beta_{1}^{(1)} \\
\beta_{1}^{(1)}
\end{array}\right] \neq\left\{\left(1-\delta_{1}\right)\left[\begin{array}{ll}
0 & 1 \\
1 & 0
\end{array}\right]+\delta_{1}\left[\begin{array}{cc}
1-a_{11} & 1-a_{12} \\
a_{11} & a_{12}
\end{array}\right]\right\}\left[\begin{array}{c}
1-\beta_{1}^{(2)} \\
\beta_{1}^{(2)}
\end{array}\right],} \\
& {\left[\begin{array}{c}
1-\beta_{1}^{(2)} \\
\beta_{1}^{(2)}
\end{array}\right] \neq\left\{\left(1-\delta_{2}\right)\left[\begin{array}{ll}
1 & 0 \\
0 & 1
\end{array}\right]+\delta_{2}\left[\begin{array}{cc}
1-a_{21} & 1-a_{22} \\
a_{21} & a_{22}
\end{array}\right]\right\}\left[\begin{array}{c}
1-\beta_{1}^{(1)} \\
\beta_{1}^{(1)}
\end{array}\right] \text {, }} \\
& {\left[\begin{array}{c}
1-\beta_{1}^{(3)} \\
\beta_{1}^{(3)}
\end{array}\right] \neq\left\{\left(1-\delta_{3}\right)\left[\begin{array}{llll}
1 & 1 & 0 & 1 \\
0 & 0 & 1 & 0
\end{array}\right]+\delta_{3}\left[\begin{array}{cccc}
1-a_{31} & 1-a_{32} & 1-a_{33} & 1-a_{34} \\
a_{31} & a_{32} & a_{33} & a_{34}
\end{array}\right]\right\} \underbrace{\left[\begin{array}{c}
\left(1-\beta_{1}^{(1)}\right)\left(1-\beta_{1}^{(2)}\right) \\
\left(1-\beta_{1}^{(1)}\right) \beta_{1}^{(2)} \\
\beta_{1}^{(1)}\left(1-\beta_{1}^{(2)}\right) \\
\beta_{1}^{(1)} \beta_{1}^{(2)}
\end{array}\right]}_{\bar{\beta}_{1}^{(1)} \otimes \bar{B}_{1}^{(2)}}}
\end{aligned}
$$

where $a_{i j}$ is a binary decision variable. From (9)-(11), we can obtain

$$
\begin{aligned}
& \left(\bar{\alpha}_{1}^{(2)}\right)^{\mathrm{T}}\left[\begin{array}{rrr}
-1 & 1 & 0 \\
0 & 0 & 1
\end{array}\right]\left[\begin{array}{c}
\delta_{1} \\
\delta_{1} a_{11} \\
\delta_{1} a_{12}
\end{array}\right]=\alpha_{1}^{(1)}+\alpha_{1}^{(2)}-1, \\
& \left(\bar{\alpha}_{1}^{(1)}\right)^{\mathrm{T}}\left[\begin{array}{rrr}
0 & 1 & 0 \\
-1 & 0 & 1
\end{array}\right]\left[\begin{array}{c}
\delta_{2} \\
\delta_{2} a_{21} \\
\delta_{2} a_{22}
\end{array}\right]=\alpha_{1}^{(2)}-\alpha_{1}^{(1)},
\end{aligned}
$$




$$
\left(\bar{\alpha}_{1}^{(1)} \otimes \bar{\alpha}_{1}^{(2)}\right)^{\mathrm{T}}\left[\begin{array}{rrrrr}
0 & 1 & 0 & 0 & 0 \\
0 & 0 & 1 & 0 & 0 \\
-1 & 0 & 0 & 1 & 0 \\
0 & 0 & 0 & 0 & 1
\end{array}\right]\left[\begin{array}{c}
\delta_{3} \\
\delta_{3} a_{31} \\
\delta_{3} a_{32} \\
\delta_{3} a_{33} \\
\delta_{3} a_{34}
\end{array}\right]=\alpha_{1}^{(3)}-\alpha_{1}^{(1)}\left(1-\alpha_{1}^{(2)}\right) .
$$

From (12)-(14), we can obtain

$$
\begin{aligned}
& \left(\bar{\beta}_{1}^{(2)}\right)^{\mathrm{T}}\left[\begin{array}{rrr}
-1 & 1 & 0 \\
0 & 0 & 1
\end{array}\right]\left[\begin{array}{c}
\delta_{1} \\
\delta_{1} a_{11} \\
\delta_{1} a_{12}
\end{array}\right] \neq \beta_{1}^{(1)}+\beta_{1}^{(2)}-1, \\
& \left(\bar{\beta}_{1}^{(1)}\right)^{\mathrm{T}}\left[\begin{array}{rrr}
0 & 1 & 0 \\
-1 & 0 & 1
\end{array}\right]\left[\begin{array}{c}
\delta_{2} \\
\delta_{2} a_{21} \\
\delta_{2} a_{22}
\end{array}\right] \neq \beta_{1}^{(2)}-\beta_{1}^{(1)}, \\
& \left(\bar{\beta}_{1}^{(1)} \otimes \bar{\beta}_{1}^{(2)}\right)^{\mathrm{T}}\left[\begin{array}{rrrrr}
0 & 1 & 0 & 0 & 0 \\
0 & 0 & 1 & 0 & 0 \\
-1 & 0 & 0 & 1 & 0 \\
0 & 0 & 0 & 0 & 1
\end{array}\right]\left[\begin{array}{c}
\delta_{3} \\
\delta_{3} a_{31} \\
\delta_{3} a_{32} \\
\delta_{3} a_{33} \\
\delta_{3} a_{34}
\end{array}\right] \neq \beta_{1}^{(3)}-\beta_{1}^{(1)}\left(1-\beta_{1}^{(2)}\right) .
\end{aligned}
$$

From the condition (ii) in Problem 2, at least one of (17)-(19) must be satisfied. Noting this fact, from (17)-(19) we can obtain

$$
\begin{aligned}
& \left(\bar{\beta}_{1}^{(2)}\right)^{\mathrm{T}}\left[\begin{array}{rrr}
-1 & 1 & 0 \\
0 & 0 & 1
\end{array}\right]\left[\begin{array}{c}
\delta_{1} \\
\delta_{1} a_{11} \\
\delta_{1} a_{12}
\end{array}\right]+1-\beta_{1}^{(1)}-\beta_{1}^{(2)}=\delta_{1,1}-\delta_{1,2}, \\
& \left(\bar{\beta}_{1}^{(1)}\right)^{\mathrm{T}}\left[\begin{array}{rrr}
0 & 1 & 0 \\
-1 & 0 & 1
\end{array}\right]\left[\begin{array}{c}
\delta_{2} \\
\delta_{2} a_{21} \\
\delta_{2} a_{22}
\end{array}\right]-\beta_{1}^{(2)}+\beta_{1}^{(1)}=\delta_{2,1}-\delta_{2,2}, \\
& \left(\bar{\beta}_{1}^{(1)} \otimes \bar{\beta}_{1}^{(2)}\right)^{\mathrm{T}}\left[\begin{array}{rrrrr}
0 & 1 & 0 & 0 & 0 \\
0 & 0 & 1 & 0 & 0 \\
-1 & 0 & 0 & 1 & 0 \\
0 & 0 & 0 & 0 & 1
\end{array}\right]\left[\begin{array}{c}
\delta_{3} \\
\delta_{3} a_{31} \\
\delta_{3} a_{32} \\
\delta_{3} a_{33} \\
\delta_{3} a_{34}
\end{array}\right]-\beta_{1}^{(3)}+\beta_{1}^{(1)}\left(1-\beta_{1}^{(2)}\right)=\delta_{3,1}-\delta_{3,2},
\end{aligned}
$$

where $\delta_{i, 1}, \quad \delta_{i, 2}, i=1,2,3$ are additional binary variables satisfying the two constraint conditions

$$
\delta_{i, 1}+\delta_{i, 2} \leq 1, \sum_{i=1}^{3} \delta_{i, 1}+\delta_{i, 2} \geq 1 .
$$

Under these conditions, at least one of $\delta_{i, 1}-\delta_{i, 2}, \quad i=1,2,3$ takes either -1 or 1 . Thus, Problem 2 for the BN in Example 2 can be rewritten as the following problem.

$$
\begin{aligned}
& \text { find } a_{11}, a_{12}, a_{21}, a_{22}, a_{31}, a_{32}, a_{33}, a_{34}, \delta_{1}, \delta_{2}, \delta_{3}, \delta_{i, 1}, \delta_{i, 2}, i=1,2,3 \text {, } \\
& \min \delta_{1}+\delta_{2}+\delta_{3},
\end{aligned}
$$

subject to (14)-(16), (20)-(22), and (23).

Finally, the product of binary variables such as $\delta_{1} a_{11}$ can be linearized by using the following lemma [27].

Lemma 1 Suppose that binary variables $\delta_{j} \in\{0,1\}, j \in \mathcal{J}$ are given, where $\mathcal{J}$ is a given index set. Then 
$\mathrm{z}=\prod_{j \in \mathcal{J}} \delta_{j}$ is equivalent to the following linear inequalities

$$
\sum_{j \in \mathcal{J}} \delta_{j}-z \leq|\mathcal{J}|-1,-\sum_{j \in \mathcal{J}} \delta_{j}+|\mathcal{J}| z \leq 0,
$$

where $|\mathcal{J}|$ is the cardinality of $\mathcal{J}$.

Using this lemma, Problem 2 for the BN in Example 2 can be rewritten as an ILP problem. From the above, we see that Problem 2 for a general BN (3) can be rewritten as an ILP problem. In general, an ILP problem is NP-hard, but several free/commercial solvers were developed. Hence, we can solve the ILP problem obtained.

\section{Example on a WNT5A Network}

In this section, as an example, we consider the gene regulatory network with the gene WNT5A, which is related to melanoma. A BN model is given by

$$
\begin{aligned}
& x_{1}(k+1)=\neg x_{6}(k), \\
& x_{2}(k+1)=\left(\neg x_{2}(k) \wedge x_{4}(k) \wedge x_{6}(k)\right) \vee\left\{x_{2}(k) \wedge\left(x_{4}(k) \vee x_{6}(k)\right)\right\}, \\
& x_{3}(k+1)=\neg x_{7}(k), \\
& x_{4}(k+1)=x_{4}(k), \\
& x_{5}(k+1)=x_{2}(k) \vee \neg x_{7}(k), \\
& x_{6}(k+1)=x_{3}(k) \vee x_{4}(k), \\
& x_{7}(k+1)=\neg x_{2}(k) \vee x_{7}(k),
\end{aligned}
$$

where the concentration level (high or low) of the gene WNT5A is denoted by $x_{1}$, the concentration level of the gene pirin by $x_{2}$, the concentration level of the gene S100P by $x_{3}$, the concentration level of the gene RET1 by $x_{4}$, the concentration level of the gene MART1 by $x_{5}$, the concentration level of the gene HADHB by $x_{6}$, and the concentration level of the gene STC2 by $x_{7}$. See [28] for further details.

For this BN model, consider solving Problem 1 . We assume that $\mathcal{A}_{d}$, and $\mathcal{A}_{u}$ are given by

$$
\begin{aligned}
& \left.\mathcal{A}_{d}=\left\{\begin{array}{lllllll}
0 & 0 & 0 & 0 & 0 & 1 & 1
\end{array}\right]^{\mathrm{T}}\right\}, \\
& \left.\mathcal{A}_{u}=\left\{\begin{array}{lllllll}
1 & 1 & 0 & 1 & 1 & 1 & 1
\end{array}\right]^{\mathrm{T}}\right\},
\end{aligned}
$$

respectively. This setting is artificially given.

First, we derive a matrix-based representation. Then, we can obtain the following matrix-based representation:

$$
\begin{aligned}
& {\left[\begin{array}{l}
x_{1}^{0}(k+1) \\
x_{1}^{1}(k+1)
\end{array}\right]=\left[\begin{array}{ll}
0 & 1 \\
1 & 0
\end{array}\right]\left[\begin{array}{l}
x_{6}^{0}(k) \\
x_{6}^{1}(k)
\end{array}\right],} \\
& {\left[\begin{array}{c}
x_{2}^{0}(k+1) \\
x_{2}^{1}(k+1)
\end{array}\right]=\left[\begin{array}{llllllll}
1 & 1 & 1 & 0 & 1 & 0 & 0 & 0 \\
0 & 0 & 0 & 1 & 0 & 1 & 1 & 1
\end{array}\right]\left[\begin{array}{c}
x_{2}^{0}(k) x_{4}^{0}(k) x_{6}^{0}(k) \\
x_{2}^{0}(k) x_{4}^{0}(k) x_{6}^{1}(k) \\
x_{2}^{0}(k) x_{4}^{1}(k) x_{6}^{0}(k) \\
x_{2}^{0}(k) x_{4}^{1}(k) x_{6}^{1}(k) \\
x_{2}^{1}(k) x_{4}^{0}(k) x_{6}^{0}(k) \\
x_{2}^{1}(k) x_{4}^{0}(k) x_{6}^{1}(k) \\
x_{2}^{1}(k) x_{4}^{1}(k) x_{6}^{0}(k) \\
x_{2}^{1}(k) x_{4}^{1}(k) x_{6}^{1}(k)
\end{array}\right],} \\
& \bar{x}_{2}(k) \otimes \bar{x}_{4}(k) \otimes \bar{x}_{6}(k)
\end{aligned}
$$




$$
\begin{aligned}
& {\left[\begin{array}{c}
x_{3}^{0}(k+1) \\
x_{3}^{1}(k+1)
\end{array}\right]=\left[\begin{array}{ll}
0 & 1 \\
1 & 0
\end{array}\right]\left[\begin{array}{l}
x_{7}^{0}(k) \\
x_{7}^{1}(k)
\end{array}\right],} \\
& {\left[\begin{array}{c}
x_{4}^{0}(k+1) \\
x_{4}^{1}(k+1)
\end{array}\right]=\left[\begin{array}{ll}
1 & 0 \\
0 & 1
\end{array}\right]\left[\begin{array}{l}
x_{4}^{0}(k) \\
x_{4}^{1}(k)
\end{array}\right] \text {, }} \\
& {\left[\begin{array}{c}
x_{5}^{0}(k+1) \\
x_{5}^{1}(k+1)
\end{array}\right]=\left[\begin{array}{llll}
0 & 1 & 0 & 0 \\
1 & 0 & 1 & 1
\end{array}\right]\left[\begin{array}{l}
x_{2}^{0}(k) x_{7}^{0}(k) \\
x_{2}^{0}(k) x_{7}^{1}(k) \\
x_{2}^{1}(k) x_{7}^{0}(k) \\
x_{2}^{1}(k) x_{7}^{1}(k)
\end{array}\right],} \\
& {\left[\begin{array}{c}
x_{6}^{0}(k+1) \\
x_{6}^{1}(k+1)
\end{array}\right]=\left[\begin{array}{llll}
1 & 0 & 0 & 0 \\
0 & 1 & 1 & 1
\end{array}\right] \underbrace{\left[\begin{array}{c}
x_{3}^{0}(k) x_{4}^{0}(k) \\
x_{3}^{0}(k) x_{4}^{1}(k) \\
x_{3}^{1}(k) x_{4}^{0}(k) \\
x_{3}^{1}(k) x_{4}^{1}(k)
\end{array}\right]}_{\bar{x}_{3}(k) \otimes \bar{x}_{4}(k)},} \\
& {\left[\begin{array}{c}
x_{7}^{0}(k+1) \\
x_{7}^{1}(k+1)
\end{array}\right]=\left[\begin{array}{llll}
0 & 0 & 1 & 0 \\
1 & 1 & 0 & 1
\end{array}\right]\left[\begin{array}{c}
{\left[\begin{array}{c}
x_{2}^{0}(k) x_{7}^{0}(k) \\
x_{2}^{0}(k) x_{7}^{1}(k) \\
x_{2}^{1}(k) x_{7}^{0}(k) \\
x_{2}^{1}(k) x_{7}^{1}(k)
\end{array}\right]} \\
\bar{x}_{2}(k) \otimes \bar{x}_{7}(k)
\end{array} .\right.}
\end{aligned}
$$

Next, we explain the solution for Problem 2. By solving this problem, we can obtain $\delta_{6}=1$ and $\delta_{i}=0$, $i=1,2,3,4,5,7$. That is, the minimum value of $|\mathcal{C}|$ is obtained by 1 , and the sets $\mathcal{P}$ and $\mathcal{C}$ are obtained by $\mathcal{P}=\{1,2,3,4,5,7\}$ and $\mathcal{C}=\{6\}$, respectively. This implies that only the Boolean function $x_{6}(k+1)=x_{3}(k) \vee x_{4}(k)$ must be replaced with other Boolean function. As the solution, we can obtain

$$
x_{6}(k+1)=x_{3}(k) \vee \neg x_{4}(k) .
$$

Finally, we observe the set of singleton attractors. For the BN model obtained, the set of singleton attractors is given by

$$
\begin{aligned}
\mathcal{A}=\left\{\left[\begin{array}{llllll}
0 & 0 & 0 & 0 & 0 & 1
\end{array}\right]^{\mathrm{T}},\left[\begin{array}{llllll}
0 & 1 & 0 & 0 & 1 & 1
\end{array}\right]^{\mathrm{T}},\left[\begin{array}{lllllll}
0 & 1 & 1 & 0 & 1 & 1 & 0
\end{array}\right]^{\mathrm{T}},\right. \\
{\left.\left[\begin{array}{lllllll}
1 & 0 & 0 & 1 & 0 & 0 & 1
\end{array}\right]^{\mathrm{T}},\left[\begin{array}{lllllll}
1 & 1 & 0 & 1 & 1 & 0 & 1
\end{array}\right]^{\mathrm{T}}\right\} . }
\end{aligned}
$$

From this set, we see that specifications on singleton attractors ( $\mathcal{A}_{d} \subseteq \mathcal{A}$ of (6) and $\mathcal{A}_{u} \cap \mathcal{A}=\varnothing$ of (7)) are satisfied.

\section{Conclusions}

For Boolean networks, we studied the problem of finding both a minimum set of control nodes and a controller, where specifications on singleton attractors are imposed. In biological networks, control nodes (control input) are not necessarily given. Hence, this problem is important in both systems biology and synthetic biology. Using a matrix-based representation of BNs, this problem can be rewritten as an integer linear programming problem. The proposed method is effective as one of the fundamental methods for control theory of gene regulatory networks.

There are several open problems. In this paper, we focused on only the set of singleton attractors. An extension of the proposed method to the case of the set of periodic attractors is important, but it will be a challenging topic. Utilizing a probabilistic BN as a model of gene regulatory networks is also important. Then, a discrete probability distribution of attractors must be considered. Finally, in order to clarify the effectiveness of the 
proposed method, it is important to consider applying the proposed method to several biological systems.

\section{Acknowledgements}

This research was partly supported by JSPS Grant-in-Aid for Scientific Research (C) 26420412.

\section{References}

[1] Santos-Rosa H. and Caldas, C. (2005) Chromatin Modifier Enzymes, the Histone Code and Cancer. European Journal of Cancer, 41, 2381-2402. http://dx.doi.org/10.1016/j.ejca.2005.08.010

[2] Kauffman, S.A. (1969) Metabolic Stability and Epigenesis in Randomly Constructed Genetic Nets. Journal of Theoretical Biology, 22, 437-467. http://dx.doi.org/10.1016/0022-5193(69)90015-0

[3] Jong, H.D. (2002) Modeling and Simulation of Genetic Regulatory Systems: A Literature Review. Journal of Computational Biology, 9, 67-103. http://dx.doi.org/10.1089/10665270252833208

[4] Akutsu, T., Hayashida, M., Ching, W.-K. and Ng, M.K. (2007) Control of Boolean Networks: Hardness Results and Algorithms for Tree Structured Networks. Journal of Theoretical Biology, 244, 670-679.

http://dx.doi.org/10.1016/j.jtbi.2006.09.023

[5] Azuma, S., Yoshida, T. and Sugie, T. (2014) Structural Monostability of Activation-Inhibition Boolean Networks. Proceedings of the 53rd IEEE Conference on Decision and Control, Los Angeles, 15-17 December 2014, 1521-1526. http://dx.doi.org/10.1109/CDC.2014.7039615

[6] Chaves, M. (2009) Methods for Qualitative Analysis of Genetic Networks. Proceedings of the European Control Conference, Budapest, 23-26 August 2009, 671-676.

[7] Cheng, D. and Qi, H. (2009) Controllability and Observability of Boolean Control Network. Automatica, 45, 16591667. http://dx.doi.org/10.1016/j.automatica.2009.03.006

[8] Kobayashi, K., Imura, J. and Hiraishi, K. (2010) Polynomial-Time Algorithm for Controllability Test of a Class of Boolean Biological Networks. EURASIP Journal on Bioinformatics and Systems Biology, 2010, Article ID: 210685.

[9] Kobayashi, K. and Hiraishi, K. (2013) Optimal Control of Gene Regulatory Networks with Effectiveness of Multiple Drugs: A Boolean Network Approach. BioMed Research International, 2013, Article ID: 246761.

[10] Kobayashi, K. and Hiraishi, K. (2014) ILP/SMT-Based Method for Design of Boolean Networks Based on Singleton Attractors. IEEE/ACM Transactions on Computational Biology and Bioinformatics, 11, 1253-1259.

[11] Langmead, C.J. and Jha, S.K. (2009) Symbolic Approaches to Finding Control Strategies in Boolean Networks. Journal of Bioinformatics and Computational Biology, 7, 323-338. http://dx.doi.org/10.1142/S0219720009004084

[12] Laschov, D. and Margaliot, M. (2009) Controllability of Boolean Control Networks via the Perron-Frobenius Theory. Automatica, 48, 1218-1223. http://dx.doi.org/10.1016/j.automatica.2012.03.022

[13] Li, F. and Sun, J. (2012) Controllability of Higher Order Boolean Control Networks. Applied Mathematics and Computation, 219, 158-169. http://dx.doi.org/10.1016/j.amc.2012.05.059

[14] Li, H., Wang, Y. and Xie, L. (2015) Output Tracking Control of Boolean Control Networks via State Feedback: Constant Reference Signal Case. Automatica, 59, 54-59. http://dx.doi.org/10.1016/j.automatica.2015.06.004

[15] Li, F. (2016) Pinning Control Design for the Stabilization of Boolean Networks. IEEE Transactions on Neural Networks and Learning Systems, 27, 1585-1590. http://dx.doi.org/10.1109/TNNLS.2015.2449274

[16] Li, H., Xie, L. and Wang, Y. (2016) On Robust Control Invariance of Boolean Control Networks. Automatica, 68, 392-396. http://dx.doi.org/10.1016/j.automatica.2016.01.075

[17] Lu, J., Zhong, J., Huang, C. and Cao, J. (2016) On Pinning Controllability of Boolean Control Networks. IEEE Transactions on Automatic Control, 61, 1658-1663. http://dx.doi.org/10.1109/TAC.2015.2478123

[18] Srihari, S., Raman, V., Leong, H.W. and Ragan, M.A. (2014) Evolution and Controllability of Cancer Networks: A Boolean Perspective. IEEE/ACM Transactions on Computational Biology and Bioinformatics, 11, 83-94. http://dx.doi.org/10.1109/TCBB.2013.128

[19] Tournier, L. and Chaves, M. (2009) Uncovering Operational Interactions in Genetic Networks Using Asynchronous Boolean Dynamics. Journal of Theoretical Biology, 260, 196-209. http://dx.doi.org/10.1016/j.jtbi.2009.06.006

[20] Shmulevich, I., Dougherty, E.R., Kim, S. and Zhang, W. (2002) Probabilistic Boolean Networks: A Rule-Based Uncertainty Model for Gene Regulatory Networks. Bioinformatics, 18, 261-274. http://dx.doi.org/10.1093/bioinformatics/18.2.261

[21] Kauffman, S.A. (1993) The Origins of Order: Self-Organization and Selection in Evolution. Oxford University Press, Oxford. 
[22] Li, H. and Wang, Y. (2012) On Reachability and Controllability of Switched Boolean Control Networks. Automatica, 48, 2917-2922. http://dx.doi.org/10.1016/j.automatica.2012.08.029

[23] Zhang, L. and Zhang, K. (2013) Controllability and Observability of Boolean Control Networks with Time-Variant Delays in States. IEEE Transactions on Neural Networks and Learning Systems, 24, 1478-1484. http://dx.doi.org/10.1109/TNNLS.2013.2246187

[24] Azuma, S., Yanagisawa, E. and Imura, J. (2008) Controllability Analysis of Biosystems Based on Piecewise Affine Systems Approach. IEEE Transactions on Automatic Control, 53, 139-152. http://dx.doi.org/10.1109/TAC.2007.911316

[25] Cheng, D., Qi, H. and Li, Z. (2011) Analysis and Control of Boolean Network: A Semi-Tensor Product Approach. Springer, Berlin. http://dx.doi.org/10.1007/978-0-85729-097-7

[26] Yices SMT Solver. http://yices.csl.sri.com/

[27] Cavalier, T.M., Pardalos, P.M. and Soyster, A.L. (1990) Modeling and Integer Programming Techniques Applied to Propositional Calculus. Computers \& Operations Research, 17, 561-570. http://dx.doi.org/10.1016/0305-0548(90)90062-C

[28] Xiao, Y. and Dougherty, E.R. (2007) The Impact of Function Perturbations in Boolean Networks. Bioinformatics, 23, 1265-1273. http://dx.doi.org/10.1093/bioinformatics/btm093

\section{Submit or recommend next manuscript to SCIRP and we will provide best service for you:}

Accepting pre-submission inquiries through Email, Facebook, LinkedIn, Twitter, etc.

A wide selection of journals (inclusive of 9 subjects, more than 200 journals)

Providing 24-hour high-quality service

User-friendly online submission system

Fair and swift peer-review system

Efficient typesetting and proofreading procedure

Display of the result of downloads and visits, as well as the number of cited articles

Maximum dissemination of your research work

Submit your manuscript at: http://papersubmission.scirp.org/ 